\title{
VEREINSRECHT
}

\section{Im Visier der Gerichte}

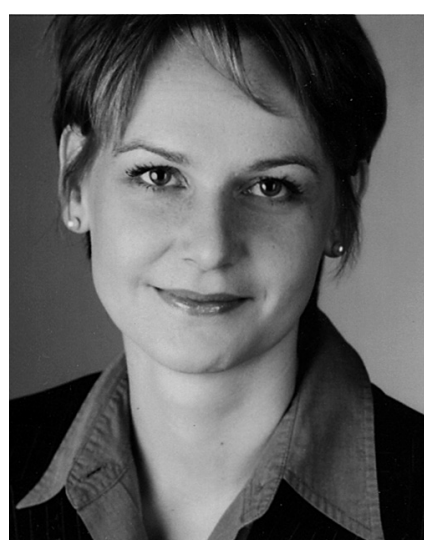

VON ERIKA KOGLIN

Erika Koglin ist Referentin für Organisationsrecht beim Paritätischen Gesamtverband in Berlin. erika.koglin@paritaet.org

\author{
In einigen Bundesländern häufen sich Fälle, in denen \\ insbesondere Kindertagesstätten in der Rechtsform \\ des eingetragenen Vereins bei Neugründungen oder \\ Satzungsänderungen von den Registergerichten die \\ Eintragung verweigert wird. Die Begründung der \\ Gerichte mit einer unternehmerischen Tätigkeit der \\ Vereine kann allerdings rechtlich nicht überzeugen.
}

»Der Idealverein ist die häufigste und typische Form des Vereins."So steht es auf der Website des Bundesjustizministeriums. (1) Das Bürgerliche Gesetzbuch (BGB) unterscheidet zwischen dem Idealverein nach $\mathbb{} 21$ BGB (dem sogenannter nicht wirtschaftlicher Verein) und dem wirtschaftlichen Verein ( $\mathbb{2} 2$ BGB). Das klingt zunächst so, als habe man die freie Wahl, als nicht wirtschaftlicher Verein nach $\mathbb{} 21$ BGB oder als wirtschaftlicher Verein nach $\$ 22$ BGB tätig zu werden.

So simpel stellt sich dies in der Praxis aber nicht dar. Denn schlägt man beispielsweise im »Palandt ", einem der wichtigsten BGB-Kommentare, nach, wie ein wirtschaftlicher Verein im Sinne des $\ 22$ BGB gegründet werden kann, so stellt man schnell fest, dass der wirtschaftliche Verein nur eine Ausnahme zu dem Idealverein ist. Die Gründung eines wirtschaftlichen Vereins ist nur zulässig, wenn es für die Vereinigung wegen der besonderen Umstände unzumutbar ist, sich als Aktiengesellschaft (AG), Gesellschaft mir begrenzter Haftung $(\mathrm{GmbH})$ oder Genossenschaft zu organisieren. Sie kann zudem nur durch besondere staatliche Verleihung erfolgen. Daher sind wirtschaftliche Vereine praktisch äußerst selten. (2) Als Argument wird aufgeführt, dass aus Gründen des Gläubigerschutzes Vereine grundsätzlich nicht wirtschaftlich tätig sein sollen. (3) Die Gründer sollen stattdessen auf die für das klassische Wirtschaftsleben vorgesehenen Rechtsformen der $\mathrm{GmbH}$, unerheblich ob als Unternehmergesellschaft oder ganz klassisch als $\mathrm{GmbH}$, oder auf die Rechtsform der Genossenschaft verwiesen werden.

Allen Vereinen ist gemein, dass der Zweck, wieso dieser Verein gegründet wurde und betrieben wird, ideeller Natur ist. Dies ist immer gleich, unabhängig davon, ob es sich um einen Sportverein, eine Kita oder um eine Selbsthilfegruppe handelt. Selbst wenn ein Verein noch ein Vereinsheim betreibt oder eine Kita, muss nach bislang geltendem Recht immer »der Zweck nicht auf einen wirtschaftlichen Geschäftsbetrieb ausgerichtet sein « - so verlangt es $\mathbb{} 21$ BGB in seiner Negativformulierung.

Maßstab für die Beurteilung ist dabei nicht nur der Wortlaut der Satzung, sondern die tatsächlich ausgeübte oder beabsichtigte Betätigung. Eine unternehmerische Betätigung ist für den Status als Idealverein dann unschädlich, wenn gemäß dem sogenannten Nebenzweckprivileg die unternehmerischen Tätigkeiten dem idealen Hauptzweck zu- und untergeordnet und Hilfsmittel zu dessen Erreichung sind. (4) Bisher galten der Betrieb von Kinderkrippen, Kindergärten und Kinderhorten ebenso wie Schulvereine unstreitig als Fälle nichtwirtschaftlicher Vereinsbetätigung. (5)

In der jüngeren Vergangenheit sorgen einige Beschlüsse des Kammergerichts Berlin aus dem Jahr 2011 für viel Wirbel und Verunsicherung in der 
Vereinslandschaft. (6) Nachdem die Zuständigkeit des Senats beim Kammergericht gewechselt hat, führte dies $\mathrm{zu}$ einer neuen Rechtsprechung hinsichtlich der Eintragungsfähigkeit von Vereinen. Kitas, aber auch Schulvereine in Berlin und Brandenburg haben seitdem Probleme mit den Registergerichten, sofern sie eine Neueintragung oder Eintragungen von Satzungsänderungen beantragen. In Brandenburg sind aber auch anlasslos Kitas zur Überprüfung ihres Zwecks angeschrieben worden, verbunden mit der Aufforderung eine andere Rechtsform zu wählen. Dies hat gravierende Auswirkungen für viele Vereine, insbesondere in Berlin, aber auch in Brandenburg, neuerdings auch vereinzelt in Rheinland-Pfalz und Baden-Württemberg.

Das Kammergericht hat in mehreren Beschlüssen zu der Abgrenzung zwischen Idealverein und wirtschaftlichem Verein Stellung genommen und die Eintragungsfähigkeit des Vereins auf seine wirtschaftliche Tätigkeit hin überprüft. (7) Es hat zum einen die Aussage getroffen, dass »der planmäßige auf Dauer angelegte entgeltliche Betrieb einer Kindertagesstätte eine entgeltliche wirtschaftliche Betätigung sei, selbst wenn nur ein kostendeckender Betrieb gewollt sei« (8) Als Indiz für die wirtschaftliche Betätigung wird die Vergütung der Vorstandsarbeit aufgeführt; Kennzeichnend für Idealvereine sei hingegen das Ehrenamt. (9) Zudem führt das Gericht aus, dass die Frage des Idealvereins losgelöst von den steuerrechtlichen Fragen der Anerkennung einer Gemeinnützigkeit zu beantworten sei. (10)

In Berlin gibt es für Elterninitiativkitas eine Besonderheit. Diese werden weiterhin durch das Registergericht eingetragen, wenn im Satzungszweck dargelegt ist, dass nicht nur die Beteiligung der Eltern gefördert wird, sondern die Eltern sich die Betreuung der Kinder teilen, indem jedes Elternteil durch Putzdienst, Kostdienst, Einkaufsdienst usw. aktiv mitarbeitet. Gefordert wird von dem Registergericht der Betrieb im reinen Ehrenamt.

Diese Auffassung der Registergerichte überzeugt nicht, da sie weder im Gesetz eine Stütze findet noch mit dem Prinzip des Nebenzweckprivilegs in Einklang steht. Zudem missachtet sie den Mitgliederwillen in dem sensiblen und verfassungsrechtlich geschützten Bereich der Vereinsautonomie.
Das Kammergericht argumentiert, dass beispielsweise Schulen in Konkurrenz zu anderen privaten Schulen mit einer Dienstleistung gegen Entgelt am Markt tätig werden würden. Dabei wird verkannt, dass der Betrieb von Schulen in der ganz überwiegenden Mehrheit keine Dienstleistung ist, die wie jede andere am Markt eingekauft werden könnte, sondern es sich hier - auch um einen staatlichen Bildungsauftrag handelt, der nicht der Gewinnerzielung dient, auch wenn theoretisch damit Geld verdient werden kann. Zudem ist jeder Einzelfall entscheidend. Es kommt nicht darauf an, wie andere beispielsweise Schulen gewerblich betreiben können.

Das Oberlandesgericht SchleswigHolstein kommt in einem ähnlich gelagerten Fall, in welchem es um den Betrieb einer Kindertagesstätte ging, zu einem anderen Ergebnis als das Kammergericht Berlin. (11) Ausgehend von der sicherlich zutreffenden Annahme, dass ideelle Zwecke kommerzialisiert werden können, kommt der Senat nicht zu dem Schluss, dass das Angebot von Leistungen zur Kinderbetreuung gegen Entgelt eine unternehmerische Tätigkeit darstelle, jedenfalls nicht in dieser Allgemeinheit im Sinne eines Automatismus. (12) Hierfür sei jeweils eine Einzelfallprüfung erforderlich. Zudem sieht das Oberlandesgericht Schleswig-Holstein den Umstand, dass die Kita gemeinnützig ist und einen entsprechenden Freistellungsbescheid vorweist als deutliches Indiz dafür an, dass in dem entschiedenen Fall die Kita in erster Linie nicht wirtschaftliche Zwecke verfolgt. (13)

Selbstverständlich gilt wie aber auch schon vor den Beschlüssen des Kammergerichts, dass der Satzungsgestaltung besondere Beachtung geschenkt werden muss und ein Verein genau überlegen sollte, welche Zwecke der Verein verwirklichen will und wie diese erreicht werden sollen und ob die Rechtsform des Vereins passend ist. Jedenfalls für Kitas und Schulvereine hat sich bislang die Rechtsform des Vereins durchaus bewährt.

Durch die angeratenen Rechtsformwechsel werden Anwälte und Berater gut verdienen, der Mitgliederwille der Vereinsmitglieder scheint dagegen kaum eine Rolle zu spielen. Das vielfach aufgeworfene Argument des Gläubigerschutzes ist zwar richtig und wichtig, jedoch kaum einschlägig, da Insolvenzen von Vereinen praktisch nicht häufig vorkommen und in der Regel sozialverträglich abgewickelt werden. Zudem kann der Gläubigerschutz durch die Rechtsform der Unternehmergesellschaft (Mini$\mathrm{GmbH}$ ) ohnehin ausgehöhlt werden. Des Weiteren besteht bei Kitas kaum ein Risiko, da diese zumeist über die öffentliche Hand refinanziert werden.

Und zuletzt: Auch die Rechtsform prägt das Geschehen in einem Zusammenschluss von Menschen. Bei einem Verein arbeite ich mit, weil ich eine Situation - auch für andere - aktiv mitgestalten will; deshalb werde ich Vereinsmitglied und fühle mich meinem Verein verbunden. Im Vordergrund steht gerade nicht das wirtschaftliche Handeln, sondern der persönliche Einsatz, unabhängig, ob dieser sich in praktischer Arbeit, in monetärer Unterstützung oder im Mitdenken niederschlägt.

\section{Anmerkungen}

(1) www.bmj.de/DE/Buerger/gesellschaft/Vereinsrecht/_doc/Idealverein.html?nn=1955982

(2) Palandt (Ellenberger), Kommentar zum BGB, 71. Auflage, $\$ 22$ Rn. 1.

(3) Reichert, Vereins- und Verbandsrecht, 12. Auflage, Rn. 118.

(4) Vgl. für viele: Sauter/Schweyer/ Waldner, 19. Auflage, Der eingetragene Verein, Rn. 47.

(5) Reichert, Vereins- und Verbandsrecht, 12. Auflage, Rn. 125; Stöber/ Otto, Handbuch zum Vereinsrecht, 10. Auflage, Rn. 79.

(6) KG Berlin, vom 18.01.2011, Az.: 25 W 14/10 und vom 21.02.2011, Az.: 25 W 32/10.

(7) Siehe Anmerkung 2, aber auch KG Berlin vom 20.01.2011, Az.: 25 W 35/10 zum Comedyfilm, KG Berlin vom 07.03.2012, Az.: 25 W. 95/11 zur Förderung der Klaviermusik

(8) KG Berlin, Beschluss vom 18.01.2011 Az.: 25 W 14/10 Rz. 13.

(9) KG Berlin, Beschluss vom 21.02.2011, Az.: 25 W 32/10. S. 5.

(10) KG Berlin, Beschluss vom 21.02.2011, Az.: 25 W 32/10. S. 5

(11) Schleswig-Holsteinisches OLG vom 18.09.2012 Az.: 2 W 152/11.

(12) Schleswig-Holsteinisches OLG vom 18.09.2012 Az.: 2 W 152/11, Rz. 44.

(13) Schleswig-Holsteinisches OLG vom 18.09.2012 Az.: 2 W 152/11, Rz. 61. 